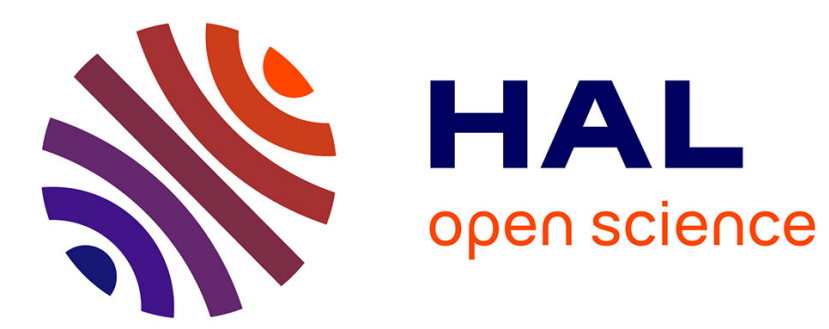

\title{
Police et pouvoir en régime de visibilité
}

Fabien Jobard

\section{To cite this version:}

Fabien Jobard. Police et pouvoir en régime de visibilité. Délibérée, 2021, La police dans tous ses états, 12, pp.19-23. halshs-03281768

\section{HAL Id: halshs-03281768 \\ https://shs.hal.science/halshs-03281768}

Submitted on 8 Jul 2021

HAL is a multi-disciplinary open access archive for the deposit and dissemination of scientific research documents, whether they are published or not. The documents may come from teaching and research institutions in France or abroad, or from public or private research centers.
L'archive ouverte pluridisciplinaire HAL, est destinée au dépôt et à la diffusion de documents scientifiques de niveau recherche, publiés ou non, émanant des établissements d'enseignement et de recherche français ou étrangers, des laboratoires publics ou privés. 
Fabien Jobard

CESDIP

fabjob@cesdip.fr

Police et pouvoir en régime de visibilité

Deux figures idéal-typiques peuvent caractériser le rapport de la police à l'État. À un pôle, la police est « le bras armé de l'État » : force dénuée d'affect et d'autonomie, la police entretient avec le pouvoir un rapport de pure instrumentalité. À l'autre pôle, la police est au contraire un "État dans l'État » : I'institution agrège tant de ressources propres qu'elle est non plus l'instrument de l'État mais, en son sein même, un corps autonome et, au besoin, dissident ou séditieux. Comme tous les idéaux-types, ces deux figures ont pour seul avantage d'aider à penser ou, en tout cas, à se représenter les choses- lesquelles, en réalité, ne se présentent jamais sous ces formes pures.

Ces deux figures-types ne conçoivent que deux entités : la police et l'État. Mais, on l'a vu ces dernières années, ce colloque singulier s'est vu maintes fois troublé par l'irruption d'une innovation démocratique que l'on ne pouvait autrefois se figurer : la massification de la caméra (et du microphone) et l'hypervisibilité de l'action policière, sa permanente publicité. Un tiers autrefois absent s'introduit : le public, qui modifie la logique même de la relation entre police et pouvoir ${ }^{1}$. II faut entendre ici " public » comme l'ensemble de celles et ceux qui sont susceptibles de voir et entendre ces images, qui deviennent, par extension, le " public » de la philosophie politique : celles et ceux qui forment l'espace public, la société, qui se fait juge des actions menées en son nom par l'État ou les élites ${ }^{2}$. Pour nourrir cette hypothèse, je partirai ici d'une actualité forte, en France, qu'est le maintien de l'ordre, parce que cette modalité d'action policière est précisément celle dans laquelle la relation entre pouvoir et police est censée être la plus rigide, la plus ordonnée, la plus simple.

Le Code de déontologie ne s'y trompe pas, qui précise (art. R. 434-10) : « Le policier ou le gendarme fait, dans l'exercice de ses fonctions, preuve de discernement. II tient compte en toutes circonstances de la nature des risques et menaces de chaque situation à laquelle il est confronté et des délais qu'il a pour agir, pour choisir la meilleure réponse légale à lui apporter ». En maintien de l'ordre au contraire, l'appréciation du sens des situations n'est pas du ressort des policiers de première ligne, ni même celle des moyens à employer pour faire cesser les troubles éventuels : tout ceci, comme l'indique le Code de la sécurité intérieure,

${ }^{1}$ Dans son ouvrage fondateur (Ce que fait la police. Sociologie de la force publique, Paris, La Découverte, 1996), Dominique Monjardet désigne « la société » comme le tiers absent du triptyque " police, pouvoir, société » (p. 230-270).

${ }^{2}$ On doit à Jürgen Habermas cette insistance sur la notion de publicité et d'espace public comme dimensions constitutives des démocraties : le spectacle de ce qui se déroule créé un public, lequel délibère sur ce qui se passe et cette délibération permet, dans une filiation kantienne de la notion, le développement de la raison et de la société politique (L'Espace public. Archéologie de la publicité comme dimension constitutive de la société bourgeoise, traduction, Paris, Payot, 1993). Dans ce qui nous intéresse, les images ne créent pas le public, qui leur pré-existe, mais leur circulation permet que la société se saisisse de ce que font les policiers et en délibèrent. 
relève de l'autorité civile ou, dans des cas bien particuliers assimilables à la légitime défense, au commandant de la force publique ${ }^{3}$.

\section{Dissimulation et transparence}

À propos de la police quotidienne, celle où le policier exerce son métier en toute autonomie, le professeur de science politique Pierre Favre pouvait écrire il y a de cela une dizaine d'années : « la police se donne une autonomie (...) puissante en raison de ses caractéristiques spécifiques (...). La police est à même de rendre étanche ses lieux d'action, de les soustraire à l'espace public, et cela autant en situation d'interpellations que dans le car de police ou dans l'enceinte du commissariat où se déroule une garde à vue ${ }^{4}$. Il insistait sur cette capacité de dissimulation de la police comme élément décuplant "l'inversion hiérarchique ", pierre angulaire du rapport de la police à l'autorité qui la commande, dans la mesure où l'autonomie des policiers sur le terrain l'emporte sur le caractère prescriptif de ce qui leur est demandé. Le policier peut toujours exciper de la singularité de la situation pour se soustraire ou plus exactement réinterpréter l’ordre qui lui a été donné.

Dix ans après l'apparition des Smartphones, la massification de l'image, qui permet aux citoyens de documenter et de tracer, en permanence, les actions menées par les policiers, place tous les choix policiers sous un régime de permanente visibilité. De moins en moins d'actions policières sont aujourd'hui soustraites aux caméras. II n'est pas jusqu'au tabassage d'un producteur de musique dans son propre studio d'enregistrement, un lieu privé, qui ne soit filmé par les caméras de vidéo-surveillance qu'il y avait installées - et vu par 20 millions de personnes, soit entre le tiers et le quart de la population française. Même les lieux ou les situations les plus propices à la soustraction de la vue du public, qu'évoquait Pierre Favre, semblent aujourd'hui accessibles au regard. Durant le confinement du printemps 2020, les mots prononcés par des policiers en pleine nuit à propos d'un homme qu'ils avaient sorti de la Seine étaient enregistrés par une caméra vidéo : " un bicot comme ça, ça nage pas $»^{5}$. Car, soulignons-le, l'image filmée est aussi une image sonore : les protagonistes parlent et se donnent à entendre en direct, dans le cours même de l'action. Les images de 152 lycéens laissés à genoux durant plusieurs heures sous le regard policiers n'auraient pas soulevé la même émotion si elles n'avaient été accompagnées de cette déclaration enjouée d'un policier : "Voilà une classe qui se tient sage ", laquelle, en une fraction de seconde, ajoute une joyeuse exubérance à l'action policière, évidemment contraire à toute déontologie.

\section{Dissimulation et transparence : l'exemple du maintien de l'ordre}

Du point de vue de l'action policière, ce que le droit a conquis en de longues et laborieuses décennies (pensons par exemple à la présence de l'avocat en garde à vue ${ }^{6}$ ), la technologie de

\footnotetext{
${ }^{3}$ Art. 431-3 du Code pénal, L211-9, D212-10 et suivants du Code de la sécurité intérieure.

${ }^{4}$ Pierre Favre, "Quand la police fabrique l'ordre social », Revue française de science politique, 59, 6, 2009, p. 1239.

${ }^{5}$ La vidéo a été relayée le 26 avril 2020 par le compte twitter de Taha Bouhafs, journaliste au site d'information en ligne Là-bas si j'y suis ". Le policier est appelé à comparaître du chef d'injures à caractère raciste le 4 mars 2021 devant le tribunal de Bobigny.

${ }^{6}$ Obtenue de haute lutte, la présence de l'avocat en garde à vue (et donc la fin de la soustraction de cette phase de l'enquête au regard extérieur) est introduite par la loi du 4 janvier 1993, qui prévoit cette présence " dès le début » de la mesure. La loi du 24 août 1993 vient d'emblée élever à 20 heures le délai au terme duquel
} 
masse l'a permis en quelques années : la visibilité, la publicité et ainsi la traçabilité de l'action des forces de police. Un tiers prend place dans la relation entre police et politique : le public, pour qui il est désormais beaucoup plus facile de demander des comptes à l'autorité politique que les faits et gestes des fonctionnaires et gendarmes lui sont connus. L'image permanente oblige davantage le politique à justifier et expliquer, sinon à réformer ou corriger, les actes des fonctionnaires et militaires qu'il investit pour maintenir l'ordre et la paix publiques. Le coût de la dissimulation policière augmente, et avec lui celui de la dénégation politique.

De cette nouvelle distribution des pouvoirs, le gouvernement paraît on ne peut mieux informé. L'augmentation du coût du déni par la massification de l'image pèse ainsi de toute sa force sur ses tentatives de nouvelles conceptions du maintien de l'ordre. L'article 24 de la loi actuellement en discussion autour de la " sécurité globale » et l'embrasement qu'il a suscité en sont une manifestation saillante ${ }^{7}$. Dans un stupéfiant déni de réalité, cet article légitime la possibilité pour la force publique d'empêcher l'image d'advenir : les dizaines et les dizaines de personnes qui filment les interventions policières, par exemple en manifestation, pourraient ainsi, au prétexte de cette nouvelle base légale, être invitées à cesser de filmer voire être interpellées et conduites en garde à vue ${ }^{8}$. Ce grotesque désir de revenir aux temps anciens de la dissimulation, s'il n'est pas balayé par la discussion parlementaire, le Conseil constitutionnel ou les instances européennes, échouera bien sûr devant son impossible mise en œuvre : le réel est toujours plus fort que la loi.

Mais une autre évolution rend bien compte du poids des images et plus de la communication dans la conduite des opérations policières. Elle est actuellement formulée dans le Schéma national du maintien de l'ordre (SNMO), adopté le 16 septembre $2020^{9}$. Ce schéma pose avec une clarté nouvelle le partage des rôles entre politique et police dans les opérations de maintien de l'ordre : le " responsable » (il est ainsi désormais nommé) est le préfet, la direction étant assumée par les chefs de police ou de gendarmerie (art. 2.41 SNMO). Et pour mieux signifier cette responsabilité politique du maintien de l'ordre, jamais aussi clairement posée que dans le SNMO, le « responsable de l'ordre public » qu'est le préfet « est présent ou se fait représenter sur le terrain (...) sur les opérations les plus complexes ou les plus sensibles ». Voir de ses propres yeux permet le commandement et, surtout, la responsabilité. On refuse peu à peu au titulaire de la décision politique le pouvoir de se soustraire, de se

\footnotetext{
le gardé à vue peut solliciter l'avocat. À la faveur d'un arrêt de la Cour européenne des droits de l'homme, la présence est à nouveau de droit dès le début de la garde à vue (art. 63-3-1 C. procéd. pén.).

${ }^{7}$ NDLR : cet article a été rédigé en décembre 2020. La proposition de loi pour la sécurité globale déposée par le député Jean-Michel Fauvergue, ancien policier, a été adoptée en première lecture à l'Assemblée nationale en novembre 2020, et devrait être examinée par le Sénat en janvier 2021 ; en l'état, l'article 24 pénalise « le fait de diffuser, par quelque moyen que ce soit et quel qu'en soit le support, dans le but manifeste qu'il soit porté atteinte à son intégrité physique ou psychique, l'image du visage ou tout autre élément d'identification, autre que son numéro d'identification individuel, d'un agent de la police nationale, d'un militaire de la gendarmerie nationale ou d'un agent de police municipale lorsque ces personnels agissent dans le cadre d'une opération de

${ }^{8}$ Il convient en effet de rappeler que l'initiative du placement en garde à vue est bien policière (article 62-2 du Code de procédure pénal), et, dans un contexte d'interpellations massives lors de manifestations, le contrôle opéré par le parquet bien souvent réduit à un pur exercice de forme.

${ }^{9}$ Consultable ici : https://www.interieur.gouv.fr/Le-ministre/Actualites/Schema-national-du-maintien-de-I-
} police. » ordre. 
dissimuler à sa responsabilité. L'ombre portée du drame de Sivens s'étend de toute évidence sur cette disposition ${ }^{10}$.

Mais ce rappel aux lois de la visibilité s'étend aujourd'hui aux manifestants eux-mêmes. Afin de dépasser les difficilement compréhensibles sommations vocales (" nous allons faire usage de la force »), éventuellement doublées du tir d'une fusée lumineuse comme le prévoit actuellement le Code de la sécurité intérieure, le même SNMO promet une politique de pure transparence : par tous moyens, les manifestants pourront voir et lire les messages, recommandations et instructions de la préfecture (art. 2.1.4). Par panneaux lumineux ou envois massifs de textos, le préfet communique avec les manifestants afin de leur indiquer ses intentions et leur signifier ses recommandations. Le manifestant, à son tour, ne peut se cacher derrière un voile d'ignorance. Et des équipes de liaison et de communication, formées à intervenir dans les manifestations et placées sous l'autorité du directeur du service d'ordre (art. 2.1.2 et 2.1.3), sont appelées à relayer ces messages auprès des manifestants, avec lesquels elles ont vocation à échanger - le tout bien sûr dans le champ des caméras et des micros, et donc sous le regard public. À l'égard de ce dernier, le gouvernement envisage de reprendre pied dans "la bataille des images ", pour reprendre l'expression du député de sa majorité, Jean-Michel Fauvergue ${ }^{11}$ et, à cette fin, accompagner, former, convaincre les journalistes pour qu'ils donnent un récit attendu des opérations menées (2.2.1 à 2.2.4).

\section{Un nouveau diagramme du pouvoir}

On mesure dans les discussions actuelles combien les tentatives de l'exécutif et du législateur de réformer le maintien de l'ordre (et peut-être la police tout entière) passent par l'image. L'agir policier est aujourd'hui placé sous le régime de la transparence et cette transparence fait émerger au sein de la relation entre police et politique, faite de dissimulations et de fauxsemblants, le tiers autrefois absent : le public. Tout indique dans la réaction actuelle du pouvoir cette importance des images dans ce nouveau " diagramme du pouvoir ", comme l'aurait sans doute nommé Michel Foucault face à ce nouveau régime de la transparence, cet omnioptique sous lequel la police agit désormais. L'exécutif, lorsqu'il ne vise pas à chasser les images comme un mauvais rêve, se plaît à imaginer pouvoir, par l'entremise de journalistes sensibilisés ou formés à cette fin, peser sur leur contenu et le commentaire qui les accompagne ; autre rêve du souverain que de dicter la chronique de son action vertueuse. S'il n'est pas certain que les rédactions acceptent cette distribution des rôles, le Schéma national du maintien de l'ordre ouvre les canaux de production d'images et de sons, facilite les échanges et la communication, en même temps qu'il prend soin de mieux identifier la ligne de partage entre ce qui relève de la décision politique et ce qui relève de l'exécution policière.

\footnotetext{
${ }^{10}$ Suite à la mort de Rémi Fraisse, tué par une grenade lancée par un gendarme dans la nuit du 25 au 26 octobre 2014 au cours de manifestations en opposition au projet de construction du barrage de Sivens, l'absence de représentant de l'autorité civile sur les lieux, alors même que des affrontements et tensions avaient été signalés, avait été largement critiquée, notamment par le Défenseur des droits (v. https://juridique.defenseurdesdroits.fr/index.php?lvl=notice_display\&id=18341).

${ }^{11} \mathrm{~J}$.-M. Fauvergue formule tout haut ce qui est une conviction profonde et ancienne chez les chefs de police, que nous relevions déjà au milieu des années 1990 : la police est contre son gré entraînée dans une guerre des images qu'elle n'a pas souhaitée et qui est menée de manière déloyale à son encontre (voir à ce propos Olivier Fillieule et Fabien Jobard, Politiques du désordre. La police et les manifestations en France, Paris, Seuil, 2020, chap. 5).
} 
La zone grise de l'action policière est frappée d'une lumière nouvelle et le politique l'a parfaitement perçu qui, du fantasme d'un monde sans image à l'échange d'égal à égal avec le citoyen manifestant, expose actuellement toutes les voies par lesquelles il envisage de vivre avec l'image et de faire vivre la police avec l'image. Un autre diagramme du pouvoir se présente, que les gouvernements peuvent utiliser de deux manières opposées. Ils peuvent choisir de se lancer, quoi qu'il en coûte du rapport à la réalité des faits, dans une bataille acharnée pour la défense de " leur " police : mener bataille après bataille une guerre pour l'imposition d'images légitimes ou de récits légitimes portant sur des images accusatrices. Choisir ainsi de se retrancher avec la police dans la défense acharnée de celle-ci, en investissant à son tour dans la production d'images, de discours ou d'alliés dans la profession journalistique et la presse. Ou bien alors gagner à lui ce tiers restauré par la transparence nouvelle de l'agir policier, qui est le public. Convaincre l'institution policière de la nécessité de sa réforme sous l'effet des images dont l'accumulation ne saurait mentir. De l'article 24 de la loi actuellement en discussion aux équipes de communication, de la prise à partie de journalistes en cours de manifestation à un "Beauvau de la sécurité ", les deux perspectives sont aujourd'hui ouvertes. Quelle que soit l'issue de cette situation neuve, le régime de la transparence policière élève si considérablement, pour le politique, les coûts de la dissimulation, qu'on a peine à l'imaginer pouvoir conserver alors son caractère démocratique. 